\title{
Solitons and Tsunamis
}

\author{
Adrian Constantin ${ }^{\mathrm{a}, \mathrm{b}}$ and David Henry ${ }^{\mathrm{a}, \mathrm{c}}$ \\ a School of Mathematics, Trinity College, Dublin 2, Ireland \\ b Current address: Universität Wien, Fakultät für Mathematik, Nordbergstraße 15, 1090 Wien, Austria \\ ${ }^{c}$ Current address: School of Mathematical Sciences, Dublin City University, Glasnevin, Dublin 9, \\ Ireland
}

Reprint requests to A. C. or D. H.; E-mail: adrian@maths.tcd.ie or hendavid@maths.tcd.ie

Z. Naturforsch. 64a, 65 - 68 (2009); received June 14, 2008

\begin{abstract}
We discuss the relevance of soliton theory to the modeling of tsunami waves. Our analysis shows that for the two most devastating tsunamis of the last century, the 2004 Bay of Bengal and the 1960 Chile tsunami, the propagation distances were too short for soliton dynamics to apply. Thus the shallow water theory is appropriate for the modelling of tsunamis.
\end{abstract}

Key words: Tsunami; Soliton; Nonlinear Waves.

PACS numbers: 47.35.Fg, 91.30.Nw, *92.10.hl

\section{Introduction}

Tsunamis are exceptionally powerful waves, generated by earthquakes, landslides, or volcanic erruptions, sweeping across deep oceans at speeds up to $800 \mathrm{~km} / \mathrm{h}$ and rising to heights of up to $30 \mathrm{~m}$ as they crash upon the shore. These extremely long waves, with distances from crest to trough of more than $100 \mathrm{~km}$, are usually not more than $1 \mathrm{~m}$ high in the deep oceans but they wreak havoc on certain coastal regions as they increase their amplitude considerably on approaching the coast.

The importance of tsunami research and analysis has grown following the catastrophic tsunami of December 26, 2004, which devastated many coastal communities around the Bay of Bengal [1,2]. The ability of predicting with greater accuracy the appearance of a tsunami and suitably preparing for its arrival depends on a better understanding of how these waves, once initiated, move, evolve and eventually become such a destructive force of nature. Two competing theories have been proposed throughout the research literature. The first is the shallow water theory, which regards tsunamis at sea as being practically linear waves, acquiring nonlinear features only as they enter shallower regions near the shore, where the bottom topography alters considerably the wave characteristics. Alternatively, it seems possible that if a tsunami propagates over large distances, in time, the individually negligible weak nonlinear factors have a significant cumulative nonlinear effect that is balanced by dis- persion to sustain waves of almost permanent form at sea, with the tsunami becoming a manifestation of solitons. The soliton theory - epitomized in the context of water waves by the Korteweg-de Vries (KdV) equation (see [3]) - represents undoubtedly one of the main achievements of nonlinear science in the last forty years. For the KdV equation an initial wave pattern evolves into a finite number of localized wave elevations (called solitons) and an oscillatory tail. Each soliton retains its identity, with taller waves traveling faster, while the oscillatory tail moves slower, disperses and spreads out in space. Therefore, in time, the wave pattern evolves into an ordered set of solitons, with the tallest in front, followed by an oscillatory tail - and the details can be predicted with great accuracy (see [3]) from knowledge of the wave pattern at some earlier instant! For the soliton theory to apply to tsunamis it is necessary that the propagation distances are long enough to allow small nonlinear effects that can be ignored at first to build up into a significant cumulative effect that is balanced by dispersion - giving thus rise to the $\mathrm{KdV}$ equation (see $[4,5]$ ). The propagation distances in the Bay of Bengal were of the order of $1600 \mathrm{~km}$ (see [2]), while the largest earthquake ever recorded, occuring in May 1960 near the Chilean coast, produced a tsunami that propagated across the Pacific Ocean and caused massive destruction in Hawaii and Japan, after traveling more than $10000 \mathrm{~km}$, respectively $17000 \mathrm{~km}$. It is therefore reasonable to regard the 1960 Chile tsunami as the best available test case 
whether soliton theory is relevant to tsunamis. We will actually assess the December 2004 tsunami and the 1960 Chile tsunami, showing that in both cases the propagation distances were too short for tsunami dynamics to apply. We conclude with a brief description of the shallow water theory approach towards the modeling of tsunamis, presenting evidence in support of it.

\section{The Relevance of Soliton Theory}

Consider a wave propagating at the surface of water in a sea with an almost flat bed of average depth $h_{0}$. Even if it originates from a storm, the typical sea wave is approximately two-dimensional [6], that is, it has a fixed propagation direction and practically no horizontal motion orthogonal to the direction of propagation. To represent such a wave, let $x$ denote the horizontal direction of propagation and let $y$ be the vertical direction, oriented upwards, with the corresponding velocity field $(u, v)$ in the fluid. Incompressibility with constant water density $\rho=1$ is a physically reasonable assumption [6], so that the equation of mass conservation takes the form

$$
u_{x}+v_{y}=0 .
$$

In the absence of strong currents we have

$$
u_{y}-v_{x}=0
$$

the flow being irrotational [7], and if $g$ is the constant acceleration of gravity and $P$ the pressure in the fluid, the equation of motion is Euler's equation

$$
\left\{\begin{array}{ccc}
u_{t}+u u_{x}+v u_{y} & = & -P_{x}, \\
v_{t}+u v_{x}+v v_{y} & = & -P_{y}-g .
\end{array}\right.
$$

Ignoring viscosity is physically realistic since the length scales required for an adjustment due to laminar viscosity are long compared to the typical wavelength encountered in sea waves [8], and the effects of friction due to turbulent mixing viscosity are negligible for sea waves that are not near the breaking stage [9]. The governing equations for water waves are obtained by supplementing $(1)-(3)$ in the fluid with appropriate boundary conditions. If $y=h(x, t)$ is the equation of the free surface, the boundary conditions are

$$
\begin{aligned}
& \left\{\begin{array}{ccc}
v=h_{t}+u h_{x} & \text { on } & y=h(x, t), \\
v=0 & \text { on } & y=0,
\end{array}\right. \\
& P=P_{0} \quad \text { on } \quad y=\eta(x, t),
\end{aligned}
$$

where $P_{0}$ stands for the constant atmospheric pressure. The conditions (4) express the fact that the free surface $y=h(x, t)$ and the flat bed $y=0$ are both interfaces, i. e., particles can not move in a transversal direction at the boundary, while the boundary condition (5) decouples the motion of the water from that of the air above it.

To predict the regions where solitons arise as accurate approximations to the surface wave it is necessary to identify the space-time region in which the $\mathrm{KdV}$ equation is an appropriate leading-order approximation of the governing equations. We now introduce the two fundamental parameters $\delta=h_{0} / \lambda$ and $\varepsilon=a / h_{0}$, where $h_{0}$ is the average water depth, $\lambda$ is the typical wavelength (larger than $150 \mathrm{~m}$ for sea waves, cf. [6]), and $a$ is the typical wave amplitude (ranging from $0.2 \mathrm{~m}$ up to $10 \mathrm{~m}$ and even larger amplitudes, cf. [6]). The KdV equation describes a balance between the effects of nonlinearity and dispersion, properties represented by the parameters $\varepsilon$ and $\delta$, respectively. It is well-known [10] that the parameter regime $\delta^{2}=O(\varepsilon)$ is suitable for the appearance of the $\mathrm{KdV}$ equation as a leading-order approximation to the governing equations in a certain spacetime region. This classical result can be obtained by defining the nondimensional free surface $\eta$ by setting $h(x, t)=h_{0}+a \eta(x, t)$ and the nondimensional pressure $p$ by setting $P=P_{0}+g\left(h_{0}-y\right)+g h_{0} p$. The nondimensionalization and scaling [11]

$$
\begin{gathered}
x \mapsto x \lambda, \quad y \mapsto y h_{0}, \quad t \mapsto t \lambda / \sqrt{g h_{0}} \\
u \mapsto \varepsilon u \sqrt{g h_{0}}, \quad v \mapsto \varepsilon v h_{0} \sqrt{g h_{0}} / \lambda, \quad p \mapsto \varepsilon p
\end{gathered}
$$

transform the governing equations (1)-(5) into the equivalent problem

$$
\left\{\begin{array}{l}
u_{t}+\varepsilon\left(u u_{x}+v u_{y}\right)=-p_{x} \quad \text { in } 0<y<1+\varepsilon \eta, \\
\delta^{2}\left[v_{t}+\varepsilon\left(u v_{x}+v v_{y}\right)\right]=-p_{y} \text { in } 0<y<1+\varepsilon \eta, \\
u_{y}-\delta^{2} v_{x}=0 \text { in } 0<y<1+\varepsilon \eta, \\
v=0 \text { on } y=0, \\
v=\eta_{t}+\varepsilon u \eta_{x} \text { and } p=\eta \quad \text { on } y=1+\varepsilon \eta .
\end{array}\right.
$$

The equations (7) lead in the regime $\delta^{2}=O(\varepsilon)$ to the $\mathrm{KdV}$ equation (see $[10,12])$ as a leading-order approximation to the governing equations for $\varepsilon \rightarrow 0$ in the region of $(x, t)$-space, where $\varepsilon t=O(1)$ and $x-t=O(1)$. Notice that setting $\delta^{2}=\varepsilon$ in (7) results in the occurrence of a single small parameter $\varepsilon$. The system (7) has a further far-reaching property: the parameter $\delta$ can be 
scaled out in favour of $\varepsilon$ as follows:

$$
\begin{aligned}
& x \mapsto x \delta / \sqrt{\varepsilon}, \quad y \rightarrow y, \quad t \mapsto t \delta / \sqrt{\varepsilon} \\
& p \mapsto p, \quad \eta \mapsto \eta, \quad u \mapsto u, \quad v \mapsto v \sqrt{\varepsilon} / \delta .
\end{aligned}
$$

The advantage of the additional scaling (8) lies in that it produces the system (7) with $\delta^{2}$ replaced by $\varepsilon$, for arbitrary $\delta$. This crucial observation makes it possible to retrieve the $\mathrm{KdV}$ equation as the appropriate leadingorder approximation to the governing equations in the region of $(x, t)$-space, where $\varepsilon t=O(1)$ and $x-t=$ $O(1)$, for any $\delta$, provided only that $\varepsilon \rightarrow 0$ (see the detailed discussion in $[4,13])$. Recalling the performed scalings and nondimensionalization (8) and (6), the region where we expect a $\mathrm{KdV}$-type balance to occur is given by $x \varepsilon \sqrt{\varepsilon} /(\delta \lambda)=O(1)$ in the original physical variables, with $t$ of the same order as $x-t \sqrt{g h_{0}}=O(1)$ in the physical variables. The interpretation of this asymptotic result is that, starting with some initial profile on the basis of which we can compute $\lambda$ and $\delta$, the $\mathrm{KdV}$ balance will occur roughly at distances of order

$$
h_{0}^{5 / 2} a^{-3 / 2}=\delta \lambda \varepsilon^{-3 / 2},
$$

from the initial disturbance. Here $a$ is the typical wave amplitude and $h_{0}$ is the typical depth, remaining roughly constant throughout this propagation distance.

Accurate measurements of the Boxing Day 2004 tsunami, provided by a radar altimeter on board of a satellite, along a track traversing the Indian Ocean/Bay of Bengal about 2 hours after the main earthquake took place are available [2]. On their basis we choose $a=$ $1 \mathrm{~m}$. On the other hand, the bathymetry of the central Bay of Bengal is relatively flat [14], with an average depth of $h_{0}=3 \mathrm{~km}$. The estimate (9) shows that a distance of about $50000 \mathrm{~km}$ is needed for the $\mathrm{KdV}$ balance, which is far in excess of the propagation distances of less than $1600 \mathrm{~km}$ from the epicentre of the earthquake to the coasts of India, Sri Lanka, or Thailand. We conclude that the propagation distances are much too short for KdV dynamics to develop on this occasion - a conclusion in agreement with other recent findings $[12,13,15]$.

The best candidate in living memory for a soliton theory approach to tsunamis is another large and destructive tsunami, triggered by the Great Chilean Earthquake on May 22, 1960 - at magnitude 9.5 on the moment-magnitude scale and with a rupture zone of over $1000 \mathrm{~km}$ the largest earthquake ever recorded $[16,17]$. The tsunami, with epicentre within $200 \mathrm{~km}$ off the coast of central Chile, propagated across the entire Pacific Ocean, reaching maximum wave heights of $10 \mathrm{~m}$ in Hawaii (after 14 hours and $10000 \mathrm{~km}$ away from the epicentre of the earthquake) and hitting with waves of $6 \mathrm{~m}$ height ( 7 hours and about $7000 \mathrm{~km}$ further away) the Japanese islands of Honsu and Hokkaido [18]. Due to the extremely long distances of propagation it was speculated $[12,15,19]$ that $\mathrm{KdV}$ dynamics were probably relevant. However, the ocean floor of the Central Pacific Basin is relatively uniform, with a mean depth of about $4300 \mathrm{~m}$. Taking $h_{0}=4.3 \mathrm{~km}$ in the above estimate for the occurrence of the $\mathrm{KdV}$ balance, for the soliton theory to become relevant within the total tsunami propagation distance of about $17000 \mathrm{~km}$, a wave amplitude exceeding $17 \mathrm{~m}$ is required. In 1960 the Pacific Tsunami Warning System was already in place and tide gauge stations throughout the Chilean coast [20] allowed for accurate measurements of the tsunami amplitudes as they hit the Chilean coastal regions shortly after initiation - these being the waves propagating east/southeast in contrast to the ones propagating in the northwest direction towards Hawaii and Japan. The average height of the wave measurements performed on the tsunami waves hitting the Chilean coast was below $10 \mathrm{~m}$ and only at 4 out of 27 locations were waves in excess of $10 \mathrm{~m}$ recorded. The particularities of the Chilean coast - with a deep sea trench about $3 \mathrm{~km}$ deep along the coast and a very gradual continental slope extending for $150 \mathrm{~km}$ from the coast to the trench [21] - greatly enhance the height of waves as they approach the shore. We conclude that a wave amplitude in excess of $17 \mathrm{~m}$ is out of question for the tsunami waves propagating in the northwest direction towards Hawaii and Japan. Thus the 1960 Chilean tsunami can not be regarded as a manifestation of the soliton theory.

\section{Conclusions}

We conclude with a brief description of the main features of the shallow water theory approach towards the modeling of tsunamis. Out in the open sea the nonlinear effects are small, so that linear long wave theory captures the main features. In particular, the waves propagate at a speed of $\sqrt{g h_{0}}$, with $g$ expressing the constant acceleration of gravity $\left(9.8 \mathrm{~m} / \mathrm{s}^{2}\right)$ - a speed proportional to the square root of the water depth [4]. In support of this theory is the fact that at the suggested speed of more than $720 \mathrm{~km} / \mathrm{h}$, corresponding 
to $h_{0}=4.3 \mathrm{~km}$, the 1960 tsunami waves originating near Chile would need about 14 hours to reach Hawaii, which is about right. Near the shore, however, the tsunami changes its nature entirely [22,23]: as depth decreases, the front of the tsunami wave slows down so the back of the wave - more than $100 \mathrm{~km}$ out at sea and approaching therefore the shore at a larger speed -

[1] V. Titov, A. B. Rabinovich, H. O. Mofjeld, R. E. Thomson, and F. I. González, Science 309, 2045 (2005).

[2] W. Craig, J. Dynam. Diff. Eq. 18, 525 (2006).

[3] P. G. Drazin and R. S. Johnson, Solitons: an Introduction, Cambridge University Press, Cambridge 1989.

[4] R.S. Johnson, A Modern Introduction to the Mathematical Theory of Water Waves, Cambridge University Press, Cambridge 1997.

[5] D. H. Sattinger, J. Math. Fluid Mech. 9, 226 (2007).

[6] J. Lighthill, Waves in Fluids, Cambridge University Press, Cambridge 1978.

[7] A. Constantin and W. Strauss, Comm. Pure Appl. Math. 57, 481 (2004).

[8] A. F. T. Da Silva and D. H. Peregrine, J. Fluid Mech. 195, 281 (1988).

[9] T. P. Barnett and K. E. Kenyon, Rep. Progr. Phys. 38, 667 (1975).

[10] M. Ablowitz and H. Segur, Solitons and the Inverse Scattering Transform, SIAM, Philadelphia 1981.

[11] With the understanding that, for example, the dimensional, physical variable $x$ is replaced by $x \lambda$, where $x$ is now a nondimensional version of the original variable.

[12] H. Segur, MSRI Publ. 55, 345 (2007) catches up. Incompressibility forces the mass of water to grow vertically so that the very long tsunami wave that was barely noticeable in the open sea becomes shorter but taller and crashes with tons of thundering water upon the shore. Near the shore nonlinear effects can not be ignored and the diminishing depth makes the shallow water theory relevant.

[13] A. Constantin and R. S. Johnson, J. Nonl. Math. Phys. 15, Suppl. 2, 58 (2008).

[14] K.V. L. N. S. Sarma, M. V. Ramana, V. Subrahmanyam, K. S. Krishna, T. Ramprasad, and M. Desa, J. Ind. Geophys. Union 4, 185 (2000).

[15] H. Segur, in: A. Kundu (Ed.), Tsunami and Nonlinear Waves, Springer, Berlin 2007, pp. 3-29.

[16] C. M. Duke, Science 132, 1797 (1960).

[17] S. Barrientos, Nature 437, 329 (2005).

[18] M. Cisternas, B.F. Atwater, F. Torrejón, Y. Sawai, G. Machuca, M. Lagos, A. Eipert, C. Youlton, I. Salgado, T. Kamataki, M. Shishikura, C.P. Rajendran, J. K. Malik, Y. Rizal, and M. Husni, Nature 437, 404 (2005).

[19] M. Lakshmanan, in: A. Kundu (Ed.), Tsunami and Nonlinear Waves, Springer, Berlin 2007, pp. 31-49.

[20] S.C. Berkman and J. M. Symons, US Department of Commerce, Coast and Geodetic Survey 1960, p. 79.

[21] R. K. Mazova and J.F. Ramirez, Natural Hazards 20, 83 (1999).

[22] A. Constantin and R. S. Johnson, J. Phys. A 39, L215 (2006).

[23] A. Constantin and R. S. Johnson, Fluid Dyn. Res. 40, 175 (2008). 